\title{
The Effects of Culture and View of Aging on Perspective Taking in Young Adults
}

\section{Christie Chung}

Department of Psychology, Mills College

ABSTRACT

Young adults' ability to recall a story about others, especially of an older adult, may be influenced by culture-based views on aging. In the present study, we extended a perspective-taking paradigm designed by Sullivan et al. (2010) by adding a cultural component to the methodology and testing participants' perspective-taking performance. Participants from the United States and Hong Kong heard two stories about a 25-year-old and a 75-year-old character from either location. Overall, we found that both groups recalled more negative than positive events. However, Hong Kong participants, who showed a more negative view of aging than US participants, used more negative words to describe the older adult than the young adult story. These findings suggest an intricate relationship between culture-based views of aging and the language used in the recall of emotional events.

\section{INTRODUCTION}

View of aging is a multi-faceted social construct that reflects beliefs on old age that goes beyond that of biological aging (Levy \& Langer, 1994; Voss et al., 2018; Yoon et al., 2000). Many studies have documented a relatively negative view of aging in the West, for example, in the US, UK, or Canada (e.g., Crockett \& Hummert, 1987; Kite \& Johnson, 1988). However, when view of aging is examined in a cross-cultural or crossnational manner, especially by comparing the West to the East, findings have been mixed (North \& Fiske, 2015). Although many studies have documented a relatively more positive view of aging in the East than the West, for example, in China, Korea, or Japan (Levy \& Langer, 1994; Chung \& Lin, 2012; Chung et al., 2014; Yoon et al., 2000), others have found a similar (e.g., Lin \& Bryant, 2009; Xiao et al., 2013) or even more negative view of aging in the East when compared to the West (e.g., Cha
\& Seo, 2009; Luo et al., 2013; Sharps et al., 1998). It has become clear that view of aging is a complex factor that would yield differential cultural differences when examined in separate domains, for example, family, work, personality, and health (Voss et al., 2018; North \& Fiske, 2015). Physical and mental abilities are in general more likely considered to decline with age, while ratings for emotional and communication skills differed more widely among cultures (Ryan et al., 2004; Harwood et al., 2001). Also, it is very important to note that countries and cultures included in the terms "Eastern" and "Western" could greatly differ within

Corresponding author: Christie Chung, Mills College Psychology Department, 5000 MacArthur Blvd., Oakland, CA 94613.

Email:cchung@mills.edu 
these groups. Therefore, it is critical that researchers take a careful approach while examining participant-level or country-level view of aging ratings.

Many studies in the psychological literature document the effects of attitudes and stereotypes on cognition. Specifically, stereotype threats and attitudes regarding aging were found to negatively affect cognitive performance in older adults (Hess, 2005). For example, Hess (2005) found that when young and older adults were primed with negative information about aging, their recall and recognition performance on a memory test was significantly worse than in participants who received less negative information. In another experiment, Hess and Hinson (2006) found that middle-aged adults exhibited a stereotype-threat lift, that is, better memory performance than young and older adults when they were presented with negative aging information before the memory test. This effect was especially strong for middle-aged adults who did not self-identify as "old." This finding suggests that age-related attitudes could significantly impact cognition, and that view of aging, which is a rather stable attitude, should be considered as an important factor when examining participants' cognitive performance. More recently, Hess et al. (2017) found that in participants aged 30 to 95, a more positive attitude toward aging was associated with an older subjective age, whereas a more positive view of self in old age was associated with younger subjective age. As subjective age or age identity could subsequently impact cognitive performance, this finding indirectly lends support to view of the influence of aging on cognition

One's subjective view of aging begins forming at a very young age and could ultimately affect how a person ages (Levy, 2009). Recent studies have found support for both environmental and genetic sources affecting view of aging development (see Kornadt \& Kandler, 2017), and Jung and Jopp (2019) also found that adult children's relationship with their parents could significantly affect how they view aging. In this study, we were particularly interested in examining the effects that culture and young adults' view of aging have on their perspective-taking ability and memory for materials related to young and older adults. Perspectivetaking is one's ability to take another person's point of view in recall of an event (Sullivan et al., 2010). Sullivan et al. (2010) designed a task where participants have to retell a story of a young or older character by taking their perspective. The authors were able to assess young and older adults' perspective-taking performance by examining the percentage of positive and negative words used in their recall performance. The idea was that participants should retell the stories based on their views and attitudes toward the developmental stage in which the character was presented. Based on this line of thinking, culture-based age-related attitudes should significantly influence perspective taking. Chung et al. (2014) demonstrated the complex relationship between culture, view of aging, and cognition by testing participants from different geographic locations and ethnic groupings. Their participants included: (a) young and older European and Chinese adults in the US (West); (b) young and older adults in Canton, China (East); and (c) Hong Kong, an international entrepôt, that was under British rule from 1897 to 1997 (East and West). In order to examine emotional memory and view of aging, participants completed an emotional picture task (Chung, 2010) and the View of Aging Task (Levy \& Langer, 1994). They found that Hong Kong young adults' view of aging scores were much more negative than those of US and mainland Chinese young adults. According to Chung et al. (2014), one explanation for this finding may be the youth-oriented culture in Hong Kong. We also believe that this finding could have been influenced by the fact that Hong Kong older adults have the longest lifespan in the world and its aging population is increasing at a rapid rate-it is characterized by an inverted aging pyramid, with mortality rate and fertility rate both rapidly decreasing in the past decades (Census and Statistics Department, 2020). However, gerontological studies have documented evidence for less than ideal welfare support for the older population, stigmatization of aging in public policy, and age discrimination in the workplace (Cheng et al., 2013; Chui et al., 2019). Children have traditionally been responsible for the care of older adults in the home, but with extreme pressures from real estate inflation and for fewer siblings to share such responsibilities, the burden that is placed on young people is tremendous (Cheng et al., 2013). Therefore, young adults' view of aging may be significantly influenced by these sociological factors. Chinese older adults in China held the most positive view of aging, followed by older adults in Hong Kong, and then Chinese immigrants and European older adults in the US. Emotional memory performance, on the other hand, did not follow the same cultural trajectory. European older adults in the US showed a positivity effect (older adults recalled positive information better than young adults), while participants in the other two groups did not. Overall, the authors reported a decoupling of view of aging and memory performance. Our follow-up question, however, was whether the relationship between view of aging and emotional memory would be different if the memory task was conducted with personally relevant materials. In the area of the positivity effect, many studies have found that the personal relevance of experimental stimuli significantly influences patterns of emotional processing. For example, using a health-related decision-making task, English and Carstensen (2015) found the positivity effect in older adults who were healthy, but not in older adults who were unhealthy. Presumably, this was because unhealthy older adults found health-related information more personally relevant compared to the healthy older adults. Gong and Fung (2020) additionally experimentally manipulated participants' ratings of personal relevance or meaningfulness of the stimuli in relation to emotional memory. Through a series of experiments, they found support for a culture-based positivity effect only when participants were specifically instructed to focus their attention on culture rather than on the emotional valence of the stimuli. Chung et al. (2014) tested emotional memory with a picture task that included pictures of scenes and objects without any human presence. Therefore, we hypothesized that if the stimuli used were changed to items or events to which the participants could personally relate, a stronger link between view of aging and memory performance might be observed.

Thus, to continue this examination of culture-bound view of aging's effect on cognition, we examined the effect that view of aging has on US and Hong Kong young adults' memory and ability to take on different age perspectives. We extended the perspective taking paradigm designed by Sullivan et al. (2010) by adding a cultural component to the method- 
ology to activate participants' culture-based view of aging-stories took place either in the US or Hong Kong. Sullivan et al. (2010) found that when young and older adults recalled stories about a 25 -year-old and a 75-year-old character, older adults used more positive and less negative language in their stories about the older than the young adult (positivity effect). However, young adults did not show an emotional difference in their retelling of stories for both characters. We hypothesized that young adults who hold different culture-based views of aging might exhibit different levels of emotional word use and memory recall. Specifically, Hong Kong young adults might use more negative words to describe the older adult story and might recall more negative events than US young adults due to their more negative view of aging, especially if the stories were presented in a self-relevant, Hong Kong context.

\section{METHOD}

\section{Participants}

A total of 95 younger adults (62 US European participants, 33 Hong Kong Chinese participants) between ages 18 and 30, without major neurological or psychiatric illnesses, participated in this study. We kept the ethnicities of our participants the same as Chung et al. (2014) for comparison purposes. Table 1 shows participants' demographics and cognitive measure scores. Independent samples t tests revealed significant differences in years of education and Trail Making Test B (Reitan, 1992) performance between US and Hong Kong participants. Therefore, we subsequently analyzed our data with these variables as covariates. Participants were recruited by email announcements and flyers at universities. Our main testing locations were Mills College (Oakland, California) and The University of Hong Kong. Both institutions utilize English as their main instructional language and all participants have learned English before the age of 7. Participants were reimbursed with extra course credit, US $\$ 10 /$ hour, or HK $\$ 80$ for participating.

\section{Materials}

Two stories (around 7 minutes each) were recorded and played as audio MP3 files on a computer. One story described events related to a 25-year- old woman and the other described events about a 75-year-old woman. Excerpts from one of the stories with positive and negative events are shown as samples below. Sullivan et al. (2010) used the same stories and conducted their analyses based on six positive and six negative events. However, after careful evaluation, we adjusted the number of emotional events for our final analyses. The younger adult story contained six positive and seven negative events, while the older adult story contained five positive events and six negative events. Due to this adjustment, we conducted our emotional memory analyses using corrected percentages of positive and negative events recalled rather than raw number of events. The stories were told in English to both US and Hong Kong participants.

An excerpt from the story about Anna, a 25-year old woman, including a positive event:

"On Friday afternoon Anna decided to make herself a cup of coffee and read the newspaper. She read articles about the upcoming elections, the dog show that would take place that weekend, and the town's high school musical that would be opening the following weekend. Her reading was interrupted when the phone rang. Answering the phone, Anna expected the caller to be someone trying to sell her carpet cleaner or dog shampoo, but was pleased when she heard the familiar voice of a friend from whom she had not heard in several years on the other end of the line. The two chatted for an hour and a half, recounting good times they had shared together and catching up on each other's lives. After saying good-bye to her friend, Anna decided to begin preparing dinner for herself."

An excerpt from the same story including a negative event:

"Since she was planning to go to a potluck the following day, Anna decided to prepare two dishes of lasagna: one lasagna that she would have some of for dinner and another lasagna that she would take to the potluck. She had just finished chopping the vegetables and soaking the noodles when she went to the cupboard and realized that she had run out of tomato sauce and forgotten to pick some up at the store the day before. This was very frustrating because she had tried to be very careful about making a complete shopping list so that she wouldn't have to go back to the store for another week, but now she was forced to head out to the store on a Friday evening."

\begin{tabular}{l} 
TABLE 1. \\
Participants' Demographics and Scores from Cognitive and Mental Status Meas \\
\hline \multicolumn{10}{c}{$\mathrm{n} \quad$} & $\begin{array}{c}\text { Age } \\
\text { (years) }\end{array}$ & $\begin{array}{c}\text { Education } \\
\text { (years) }\end{array}$ & $\begin{array}{c}\text { Trail } \\
\text { VOA }\end{array}$ & $\begin{array}{c}\text { Making } \\
\text { A (s) }\end{array}$ & $\begin{array}{c}\text { Makil } \\
\mathrm{B}(\mathrm{s})\end{array}$ & $\begin{array}{c}\text { Digit } \\
\text { Symbol } \\
\text { Encoding } \\
(\mathrm{s})\end{array}$ \\
\hline
\end{tabular}

Note. SDs are given in parentheses. View of aging (VOA) rating scale: $0=n e g a-$ tive, $0.5=$ neutral, $1=$ positive.

${ }^{* *} p<.01$ 


\section{Procedure}

After giving informed consent and completing a health and demographics questionnaire, participants listened to the stories described above. Each participant heard both the young and older character stories with presentation order counterbalanced. Before presentation of each story, participants were told four facts: the character's name (either Anna or Emma), where the character was from (either US or Hong Kong), how old the character was (either 25 or 75 years old), and that they would be asked to recall the story in detail when it ended. Participants completed the Trail-Making Task (Reitan, 1992) after listening to the first story (25- or 75-year old). After this task was completed, participants were instructed to recall out loud as much detail as they could remember from the story in the perspective of the main character. Their verbal responses were recorded on the computer. Participants were given as much time as needed to recall the story in detail. After this testing phase, participants heard the second story (i.e., about the person from the other age group), which took place in the same location as the first story. The Digit Symbol Task (Salthouse, 1992) was administered before participants recalled the second story. In a follow-up study, participants completed the View of Aging Task (Yoon et al., 2000), which required them to state five words or phrases that came to mind when they thought of old age.

\section{RESULTS}

\section{View of Aging}

Two independent raters who were unrelated to the study evaluated the view of aging results ( $\alpha=.87$ ) using the following rating scale by Yoon et al. (2000): $0=$ negative, $0.5=$ neutral, $1=$ positive. An independentsamples $t$ test revealed a significant difference in view of aging ratings between the US $(M=.33)$ and Hong Kong participants $(M=.23), t=$ $2.54, p=.013, d=.60$ (see Table 1$)$.

\section{EMOTIONAL WORDS IN PERSPECTIVE TAKING TASK}

Participants' recall narratives were analyzed using the Linguistic Inquiry and Word Count (LIWC, Pennebaker et al., 2015) program for percentage of emotional word use (positive and negative). A mixedfactor $2 \times 2 \times 2 \times 2$ (testing location [US, HK] $\times$ character age [older, younger $\times$ character nationality [US, HK $\times$ word valence [positive, negative]) analysis of variance (ANOVA) was conducted with testing location and character nationality as a between-group variables and the rest as within-group variables. Our results revealed a significant three-way interaction among testing location, character age, and word valence, $F(1,90)=5.25, p=.024, \eta^{2}=.055$. The main effect of valence was also significant, $F(1,90)=11.73, p=.001, \eta^{2}=.115$, with participants from both locations using more positive $(M=1.78 \%)$ than negative words $(M=1.31 \%)$. These results remained consistent when education and Trail Making Test B performance were added as covariates to an ANCOVA analysis.

Post-hoc $t$ tests showed that Hong Kong participants used significantly more negative words in their recall of the older adult $(M=$ $1.69 \%)$ than the young adult story $(M=.94 \%)$, regardless of character nationality, $t(31)=2.77, p=.009, d=.74$. When data were separated by location, we found that US participants used more positive $(M=$ $1.75 \%)$ than negative words $(M=1.24 \%)$ to describe the older adult story regardless of character nationality, $t(61)=4.02, p<.001, d=.69$, while Hong Kong participants used more positive $(M=1.86 \%)$ than negative words $(M=.94 \%)$ in their recall of the young adult story of both nationalities, $t(31)=4.02, p<.001, d=1.05$. Means and standard errors for positive and negative words used in participants' recall are displayed in Figure 1.

We explored the relationship between our participants' view of aging ratings and emotional word use in memory recall. A correlation analysis conducted separately for US and Hong Kong participants revealed a significant positive correlation between view of aging ratings and positive word use in the recall of the older character story by Hong Kong participants, $r=.37, p=.036$.

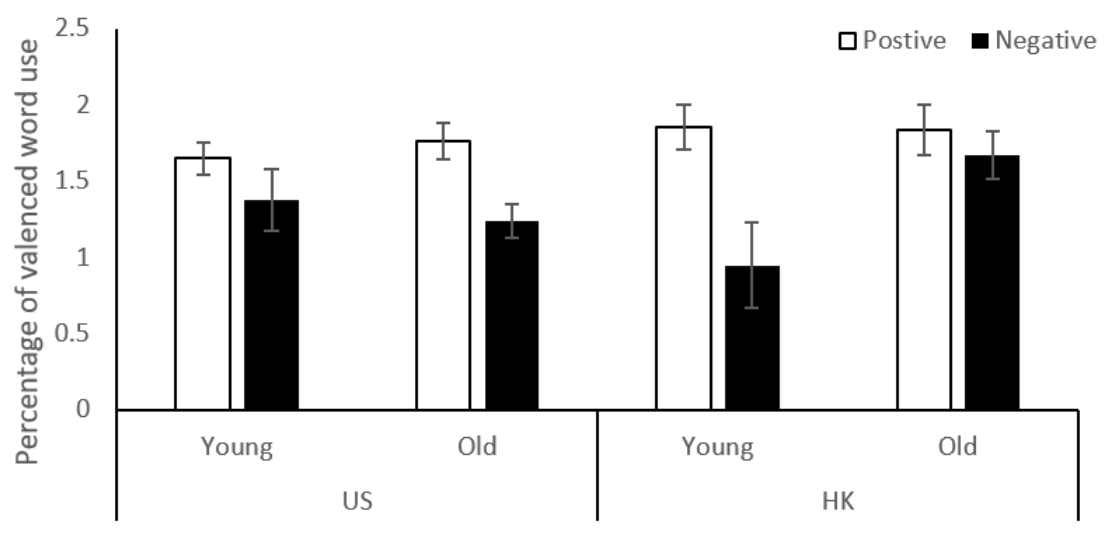

Testing Location

FIGURE 1.

Percentages of positive and negative words used by US and Hong Kong young participants. Error bars represent standard errors. 


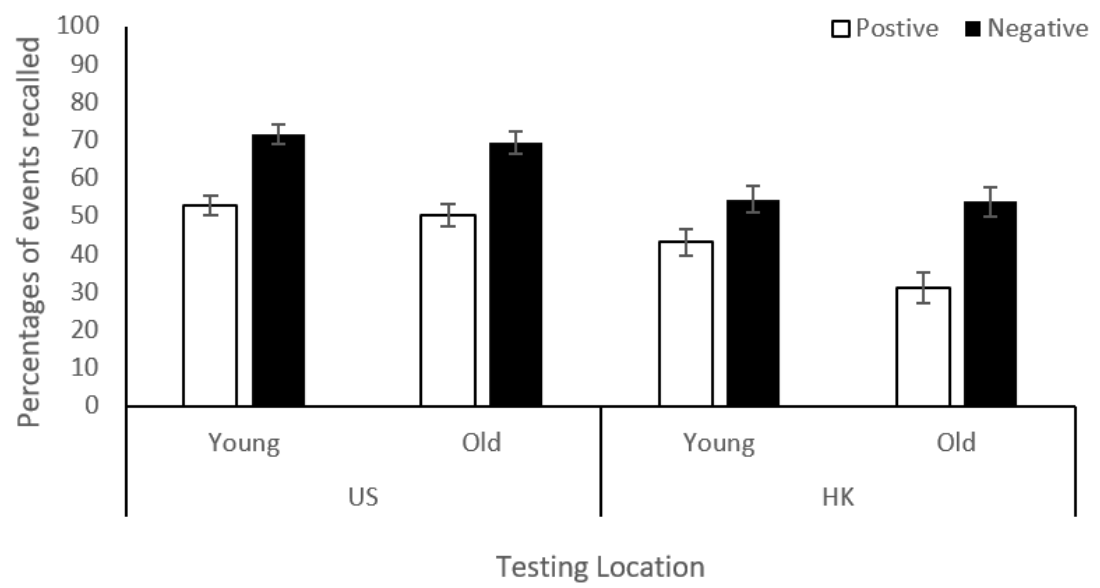

FIGURE 2.

Percentages of emotional events recalled by US and Hong Kong young participants. Error bars represent standard errors.

\section{EMOTIONAL EVENTS RECALLED}

Participants' recall memory was also evaluated for emotional valence by two independent raters $(\alpha=90)$. Analyses were conducted on the percentage of positive and negative events each participant recalled from the stories. A mixed-factor $2 \times 2 \times 2 \times 2$ (testing location [US, $\mathrm{HK}$ ] $\times$ Age [older, younger] $\times$ character nationality [US, HK] $\times$ event valence [positive, negative]) ANOVA revealed a significant main effect of age, $F(1,90)=6.10, p=.015, \eta^{2}=.063$, where participants recalled more events for the young $(M=55.60 \%)$ rather than the older character $(M=51.27 \%)$. The main effect for event valence was also significant, $F(1,90)=80.90, p<.001, \eta^{2}=.473$. Overall, participants recalled more negative $(M=62.38 \%)$ than positive events $(M=44.48 \%)$. This ANOVA also revealed a significant main effect for testing location, $F(1$, $90)=20.24, p<.001, \eta^{2}=.18$, with participants in the US $(M=61.11 \%)$ recalling more events than Hong Kong participants $(M=45.75 \%)$. Other effects were either marginal or nonsignificant.

\section{DISCUSSION}

In the present study, we examined the effects of view of aging and culture on perspective taking and memory. We added a cultural component to a paradigm designed by Sullivan et al. (2010) and tested young participants from the US and Hong Kong. We collected view of aging data from all participants and examined the percentages of emotional events recalled and emotional word use in their memory recall. Sullivan et al. (2010) found a positivity effect in perspective taking, in that older participants used more positive words and fewer negative words in their recall of the older adult story than in the young adult story. However, young participants in their study did not show any emotional difference in their linguistic recall. Our study replicated the US young adult portion of their findings, and extended it by demonstrating a negativity bias in word use toward older adults in the Hong Kong sample. Also, young adults in both US and Hong Kong recalled a higher percentage of negative than positive events, which replicates
Sullivan et al's (2010) findings and supports the negativity bias literature in cognition (e.g., Baumeister et al., 2001; Cacioppo et al., 1997; Wood \& Kisley, 2006). Overall, Hong Kong participants demonstrated a significantly more negative view of aging than US participants, which could have contributed to their negativity bias toward the older character.

The finding of Hong Kong young adults exhibiting a more negative view of aging is in line with Chung et al. (2014), where the authors found Hong Kong young adults' view of aging ratings to be significantly more negative than those of US and mainland Chinese participants. Explanations for this finding may be the youth-oriented culture as mentioned in Chung et al. (2014), plus the rapid burgeoning of the older population in Hong Kong but less than ideal cultural and societal support for the aging population, as discussed in the Introduction section (see also Cheng et al., 2013). However, the current study adds to the existing literature by demonstrating a link between view of aging and cognitive performance by examining how the view of an age group may contribute to cognitive performance for information related to that group. In our perspective-taking task, we see a clear negativity bias in Hong Kong young adults' word use in recalling the older adult story, but not the young adult story. Hong Kong participants used significantly more negative words to describe the older than the young adult story, while US participants did not show this pattern of results. Further, US participants used more positive than negative words in their recall of the older adult story, which reflects an age-related positivity effect often found in emotional memory studies (Carstensen, 2006; Carstensen \& Mikels, 2005). Through a correlation analysis, we also found that Hong Kong participants with more positive view of aging ratings used more positive words in their recall of the older adult story. Meanwhile, US participants showed a less negative view of aging and did not exhibit the above negativity bias in the perspective taking task. This result might have been reinforced by the fact that the stories read were mostly related to friends and leisure, domains where view of aging was found to be less positive in Hong Kong than in the US 
in a study by Voss et al. (2018). Future studies could be designed to examine whether this factor truly contributed to the present findings by using stimuli that are related to domains where view of aging was found to be more positive in Hong Kong participants than in US participants, for example, personality and finances.

The present findings suggest that participants' view of aging might play an important role in perspective-taking. Recent studies in the area of emotion and culture have converged on the idea that cognition can be significantly affected by stimuli that participants find meaningful (e.g., Fung et al., 2019). This idea can be extended to explain the present findings as Hong Kong participants appeared to be significantly affected by the age of the character in each story. Chung et al. (2014) found a decoupling of view of aging and emotional memory performance with picture stimuli of scenes and objects that were unrelated to the participants and had no human beings in the stimuli at all. In contrast, the stimuli and instructions used in the present study were much more personal, as participants had to take the perspective of the story character during recall. Taken together, these findings point to the importance of personal relevance in the stimuli for view of aging to exert an effect in cognition.

Contrary to our hypothesis, character nationality did not make a difference in participants' word use and recall performance. This could be because view of aging was a powerful attitude that took precedence over other attitudes in our experimental context. We manipulated the age of the character as a within-subject factor, but their nationality was a between-subject factor, that is, all participants heard both the young and older adult story, but they were either told that the story took place in the US or in Hong Kong. Hearing both the young and older adult story could have potentially primed participants to activate more age-related attitudes rather than nationality-related attitudes. In future studies, we plan to further explore the effects of age and culture on perspective taking and memory by designing studies that would allow us to equally assess the contribution of both factors within one study. We would also be interested in extending the present study to older adults to examine these findings in relation to the positivity effect in old age (Carstensen, 2006; Carstensen \& Mikels, 2005).

We also examined participants' event memory recall and replicated the results reported by Sullivan et al. (2010). Participants from both the US and Hong Kong recalled more negative memories than positive memories. This young adult negativity bias is widely documented in the psychological literature, which suggests a general tendency for young adults to focus more on processing negative rather than neutral and positive information (e.g., Baumeister et al., 2001; Cacioppo et al., 1997; Wood \& Kisley, 2006). Our results also revealed a significant main effect of age, where participants recalled significantly more events for the young character story than the older character story. This finding can once again be explained by our discussion regarding selfrelevance and meaningfulness. It is also a demonstration of an own-age effect (Anastasi \& Rhodes, 2005), which is often observed in memory studies that utilize face recognition paradigms whereby young participants show better memory for young faces, and older participants display higher accuracy in remembering older faces. Again, these findings highlight the importance of the self-relevance nature of stimuli used in memory tasks.

At first glance, our word use and event memory analyses appear to yield discrepant patterns of results. However, it is important to note that Sullivan et al's (2010) study found a similar dissociation between word use and event memory, that is, even though they found a significant difference in emotional word use between young and older adults, the two groups did not differ in their emotional memory recall patterns. This decoupling of word use and event memory recall performance suggests that linguistic expression and emotional memory processing could be separate processes. Sullivan et al. (2010) explained that even though older adults used more positive words in their recall of a young adult story, they were not "blind" to the negativity of the events. They were simply putting the best "face" on the negative events during their recall performance. In a similar way, both our US and Hong Kong participants recalled more negative events overall, but Hong Kong adults simply described these negative events with more negative wording. This finding highlights the importance of our linguistic analysis in the study of perspective-taking. Simply examining memory recall would not have given us the insight about participants' reflection and representation of these remembered events.

Although the present study provides important additional information about the influence of culture on perspective-taking, it is not without limitations. First of all, our study was conducted in English in both the US and Hong Kong. Although all participants attend universities that utilize English as an instructional language and had all learned English before the age of 7 (a common criterion for cognitive studies), we understand our reviewers' questions about a potential difference in language processing and usage in the two samples. In fact, this language issue could have led to the lower rate of recall in our Hong Kong sample in comparison to our US sample. Therefore, in future studies, we plan to implement English vocabulary and proficiency tests in our testing procedure. Testing in participants' native languages could also be an option, although it may be difficult to conduct comparison analyses on word usage as we did in the present study.

Another limitation is that we only included female characters in the stories. Although we did not observe a significant effect of participant sex in our study, we did notice a trend for Hong Kong female participants using more positive words and fewer negative words than their male counterparts. We believe that sex and gender are essential factors to consider as we delve deeper into how self-relevant stimuli may impact perspective taking and memory performance.

The present study is an important addition to the growing field of aging attitudes and cross-cultural cognition. As people's lifespan increases significantly worldwide, it is imperative that we understand the effect of age-related views and stereotypes on cognition. Young adults will have to interact with more and more older adults on a daily basis and, therefore, it is critical to understand how age-related views may impact our cognition and behaviors and vice versa. The present study does not only extend our understanding of the effect of culture and view of aging on perspective-taking and memory, but could also be applied in settings such as hospitals, nursing homes, and social settings 
where young adults have to interact with older adults. For example, young medical professionals would benefit from the results of this study as they look after older adults in medical settings. Their view of aging could inevitably influence their interaction with older adults and affect their memory for the events that took place during medical visits. In academic settings, young students often interact with instructors who are older, and thus, understanding how view of aging may affect young adults' cognition could encourage mutual understanding to foster a collaborative learning environment. For example, young adults' culture-based negative bias toward older adults could extend to the area of technology and online communication, and as virtual learning becomes a part of modern education, young adults' biases toward older instructors could greatly affect their learning experience and the way they evaluate their instructors at the end of the semester. The current results may also be useful for marketing purposes as advertisers make decisions on the age and ethnicity of models used in representing their brands both locally and globally, as consumers may retain information about a product better if the models are personally relevant to them. Future studies could aim to replicate the present study in applied settings, preferably with participants from different age groups, to further understand the effects of culture and view of aging on cognition throughout the lifespan.

\section{REFERENCES}

Anastasi, J. S., \& Rhodes, M. G. (2005). An own-age bias in face recognition for children and older adults. Psychological Bulletin Review, 12(6), 1043-1047. https://doi.org/10.3758/BF03206441 المسلسلسا

Baumeister, R. F., Bratslavsky, E., Fickenauer, C., \& Vohs, K. D. (2001). Bad is stronger than good. Review of General Psychology, 5, 323-370. https://doi.org/10.1037/1089-2680.5.4.323 |لس

Cacioppo, J. T., Gardner, W. L., \& Berntson, G. G. (1997). Beyond bipolar conceptualizations and measures: The case of attitudes and evaluative space. Personality and Social Psychology Review, 1, 3-25. https://doi.org/10.1207/s15327957pspr0101_2 الملسلسلس

Carstensen, L. L. (2006). The influence on a sense of time on human development. Science, 312, 1913-1915. https://doi.org/10.1126/science.1127488 الس الس

Carstensen, L. L., \& Mikels, J. A. (2005). At the intersection of emotion and cognition: Aging and the positivity effect. Current Directions in Psychological Science, 14, 117-121. https://doi.org/10.1111/j.09637214.2005.00348.x سلس

Census and Statistics Department, HKSAR. (2020, October). Hong Kong statistics. http://censtatd.gov.hk/hkstat/

Cha, M., \& Seo, S. (2009). A cross-country exploration: Dietetic students' knowledge, attitudes, and intentions to provide services to the elderly. Educational Gerontology, 35, 801-817. https://doi. org/10.1080/03601270902717996 الملسلس

Cheng, S-T., Lum, T., Lam, L. C. W., \& Fung, H. H. (2013). Hong Kong: Embracing a fast aging society with limited welfare. The Gerontologist, 53, 527-533.

Chui, C. H-K., Tang, J. Y. M., Kwan, C. M., Chan, O. F., Tse, M., Chiu, R. L. H., Lou, V. W. Q., Chau, P. H., Leung, A. Y. M., \& Lum, T. Y. S.
(2019). Older adults' perceptions of age-friendliness in Hong Kong. The Gerontologist, 59, 549-558. https://doi.org/10.1093/geront/gny052 السلسلسلسال Chung, C. (2010). Effects of view of life and selection bias on emotional memory in old age. GeroPsych: The Journal of Gerontopsychology and Geriatric Psychiatry, 23, 161-168. https://doi.org/10.1024/16629647/a000019 الس الس

Chung, C., \& Lin, Z. (2012). A cross-cultural examination of the positivity effect in memory: United States vs. China. International Journal of Aging and Human Development, 75, 31-44. https://doi. org/10.2190/AG.75.1.d 面

Chung, C., Yip, A., \& Lin, Z. (2014). Culture, attitudes, and emotional memory: A Complicated relationship. International Journal of Research Studies in Psychology, 3, 1-12. https://doi.org/10.5861/ ijrsp.2014.866 السلسلسلس

Crockett, W.H., \& Hummert, M.L. (1987). Perceptions of aging and the elderly. Annual Review of Gerontology and Geriatric, 7, 217-241. المالسلس

English, T., \& Carstensen, L. L. (2015). Does positivity operate when the stakes are high? Health status and decision making among older adults. Psychology and Aging, 30, 348-355. https://doi.org/10.1037/ a0039121 الفلسلس

Fung, H. H., Lu, M., \& Isaacowitz, D. M. (2019). Aging and attention: Meaningfulness may be more important than valence. Psychology and Aging, 34, 85-90. https://doi.org/10.1037/pag0000304 المالبلسا

Gong, X., \& Fung, H. H. (2020). Remembering positive or relevant information? Cultural relevance may moderate the age-related positivity effect in memory. Psychology and Aging, 35, 267-282. https:// doi.org/10.1037/pag0000438 المالسلسلة

Harwood, J., Giles, H., McCann, R. M., Cai, D., Somera, L. P., Ng, S. H., Gallois, C., \& Noels, K. (2001). Older adults' trait ratings of three agegroups around the Pacific rim. Journal of Cross-Cultural Gerontology, 16, 157-171. https://doi.org/10.1023/A:1010616316082 الملسلس

Hess, T. M. (2005). Memory and aging in context. Psychological Bulletin,

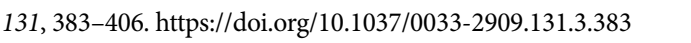

Hess, T. M., \& Hinson, J. T. (2006). Age-related variation in the influences of aging stereotypes on memory in adulthood. Psychology and Aging, 21, 621-625. https://doi.org/10.1037/0882-7974.21.3.621 المبلسلكسل Hess, T. M., O'Brien, E. L., Voss, P., Kornadt, A. E., Rothermund, K., Fung, H. H., \& Popham, L. E. (2017). Context influences on the relationship between views of aging and subjective age: The moderating role of culture and domain of functioning. Psychology and Aging, 32, 419-431. https://doi.org/10.1037/pag0000181 السلسلس

Jung, S., \& Jopp D. S. (2019). Adult children's relationship top parent influences their views on aging and attitude toward own aging. The International Journal of Aging and Human Development, 89, 231256. https://doi.org/10.1177/0091415018784703 الس الس

Kornadt, A. E., \& Kandler, C. (2017). Genetic and environmental sources of individual differences in views on aging. Psychology and Aging, 32, 388-399. https://doi.org/10.1037/pag0000174 سلس

Kite, M. E., \& Johnson, B. T. (1988). Attitudes toward older and younger adults: A meta-analysis. Psychology and Aging, 3, 233-244. https://doi.org/10.1037/0882-7974.3.3.233 سلس

Levy, B. (2009). Stereotype embodiment: A psychosocial approach 
to aging. Current Directions in Psychological Science, 18, 332-336. https://doi.org/10.1111/j.1467-8721.2009.01662.x الس السلس

Levy, B., \& Langer, E. (1994). Aging free from negative stereotypes: Successful memory in China and among the American deaf. Attitudes and Social Cognition, 66, 989-997. https://doi. org/10.1037/0022-3514.66.6.989 الس السلس

Lin, X., \& Bryant, C. (2009). Students' attitudes toward older people: A cross-cultural comparison. Journal of Intergenerational Relationships, 7, 411-424. https://doi.org/10.1080/15350770903285320 الس السلسل|

Luo, B., Zhou, K., Jin, E. J., Newman, A., \& Liang, J. (2013). Ageism among college students: A comparative study between U.S. and China. Journal of Cross-Cultural Gerontology, 28, 49-63. https://doi. org/10.1007/s10823-013-9186-5 الم السلسلس

North, M. S., \& Fiske, S. T. (2015). Modern attitudes toward older adults in the aging world: A cross-cultural meta-analysis. Psychological Bulletin, 141, 993-1021. https://doi.org/10.1037/a0039469 سلس

Pennebaker, J. W., Boyd, R. L., Jordan, K., \& Blackburn, K. (2015). The development and psychometric properties of LIWC2015. المالسلسل

Reitan, R. M. (1992). Trail Making Test: Manual for administration and scoring. South Tucson, AZ: Reitan Neuropsychology Laboratory.

Ryan, E. B., Jin, Y., Anas, A. P., \& Luh, J. J. (2004). Communication beliefs about young and old age in Asia and Canada. Journal of Cross-Cultural Gerontology, 19, 343-360. https://doi.org/10.1023/ B:JCCG.0000044688.27282.7b السلسلس

Salthouse, T. A. (1992). Influence of processing speed on adult age differences in working memory. Acta Psychologica, 79, 155-170. https://doi.org/10.1016/0001-6918(92)90030-H المالسلس الس الس
Sharps, M. J., Price-Sharps, J. L., \& Hanson, J. (1998). Attitudes of young adults toward older adults: Evidence from the United States and Thailand. Educational Gerontology, 24, 655-660. https://doi. org/10.1080/0360127980240703 الس الس سلس

Sullivan, S. J., Mikels, J. A., \& Carstensen, L. L. (2010). You never lose the ages you've been: Affective perspective taking in older adults. Psychology and Aging, 25, 229-234. https://doi.org/10.1037/a0018383 السلسلس

Xiao, L. D., Shen, J., \& Paterson, J. (2013). Cross-cultural comparison of attitudes and preferences for care of the elderly among Australian and Chinese nursing students. Journal of Transcultural Nursing, 24, 408-416. https://doi.org/10.1177/1043659613493329 المالسلسل|

Voss, P., Kornadt, A. E., Hess, T. M., Fung, H. H., \& Rothermund, K. (2018). A world of difference? Domain-specific views on aging in China, the US, and Germany. Psychology and Aging, 33, 595-606. https://doi.org/10.1037/pag0000237 المالسلسل

Wood, S., \& Kisley, M. A. (2006). The negativity bias is eliminated in older adults: Age-related reduction in event-related brain potentials associated with evaluative categorization. Psychology and Aging, 2, 815-820. https://doi.org/10.1037/0882-7974.21.4.815 الس

Yoon, C., Hasher, L., Feinberg, F., Rahhal, T. A., \& Winocur, G. (2000). Cross-cultural differences in memory: The role of culture-based stereotypes about aging. Psychology and Aging, 15, 694-704. https:// doi.org/10.1037/0882-7974.15.4.694 المالسلسا 